\title{
Extension to imaginary chemical potential in a holographic model
}

\author{
Kazuo Ghoroku๑, ${ }^{1, *}$ Kouji Kashiwa $\odot,{ }^{1, \dagger}$ Yoshimasa Nakano $\odot,{ }^{*}$ Motoi Tachibana, ${ }^{2, \S}$ and Fumihiko Toyoda ${ }^{3, \|}$ \\ ${ }^{1}$ Fukuoka Institute of Technology, Wajiro, Fukuoka 811-0295, Japan \\ ${ }^{2}$ Department of Physics, Saga University, Saga 840-8502, Japan \\ ${ }^{3}$ Faculty of Humanity-Oriented Science and Engineering, Kinki University, Iizuka 820-8555, Japan
}

(Received 1 June 2020; accepted 16 July 2020; published 5 August 2020)

\begin{abstract}
We extend a bottom-up holographic model, which has been used in studying the color superconductivity in QCD, to the imaginary chemical potential $\left(\mu_{I}\right)$ region, and the phase diagram is studied on the $\mu_{I}$-temperature $(T)$ plane. The analysis is performed for the case of the probe approximation and for the background, where the backreaction from the flavor fermions is taken into account. For both cases, we could find the expected Roberge-Weiss (RW) transitions. In the case of the backreacted solution, a bound of the color number $N_{c}$ is found to produce the RW periodicity. It is given as $N_{c} \geq 1.2$. Furthermore, we could assure the validity of this extended model by comparing our result with that of the lattice QCD near $\mu_{I}=0$.
\end{abstract}

DOI: $10.1103 /$ PhysRevD.102.046003

\section{INTRODUCTION}

The holographic approach based on the string/gauge duality is a powerful method to study various thermodynamical and nonperturbative properties of the Yang-Mills theory, especially when the chemical potential $(\mu)$ of the fundamental fermions plays an important role. Various results have been obtained in such cases, and they could give us many kinds of insight into the phase diagram of QCD. (See, for example, Refs. [1-4].)

On the other hand, many nonperturbative investigations in QCD have been performed by the lattice gauge theory. However, the analysis has been restricted to the case of the imaginary chemical potential, $\mu_{I}$, to avoid the sign problem of the fermion determinant (See, for example, Refs. [5,6]). In QCD with $\mu_{I}$, on the other hand, the Roberge-Weiss (RW) phase transition and its periodicity with respect to $\mu_{I}$ have been pointed out as a remarkable point [7]. This observation is understood from the periodicity of the partition function. It would be meaningful to see how this point is realized in the holographic approach to make clear the validity of the holographic approach.

Ten years ago, however, such a holographic investigation was made based on the Euclidean space-time geometry

\footnotetext{
*gouroku@fit.ac.jp

†kashiwa@fit.ac.jp

*ynakano@kyudai.jp

\$motoi@cc.saga-u.ac.jp

"f1toyoda@jcom.home.ne.jp
}

Published by the American Physical Society under the terms of the Creative Commons Attribution 4.0 International license. Further distribution of this work must maintain attribution to the author(s) and the published article's title, journal citation, and DOI. Funded by SCOAP .
[8-10]. In Ref. [8], the periodic RW transition has been shown by adding the two-form Kalb-Ramond field $B$ in the D3/D7-brane system of the type-IIB model. After that, and also in the D4/D8-brane system in the IIA model, similar analysis has been done in Ref. [9], and also in Refs. [10,11] in a slightly different method. In these approaches, the essential point is the introduction of the $B$ field with $d B=0$ and its potential $V_{A}(\alpha)$, where $\alpha$ corresponds to the phase of the Polyakov loop $[12,13]$. It is introduced as ${ }^{1}$

$$
\alpha=\int_{D_{2}} \frac{B}{2 \pi \alpha^{\prime}} .
$$

And this parameter $\alpha$ discriminates the periodic vacua in the deconfinement phase with spontaneously broken $Z_{N}$ symmetry. On the other hand, $\mu_{I}$ comes from the bulk U(1) gauge field $F\left(=F_{\mu \nu} d x^{\mu} \wedge d x^{\nu}\right)$, and it appears in the theory being combined with $\alpha$ as

$$
\alpha-\frac{\mu_{I}}{T}=\int_{D_{2}}\left(F+\frac{B}{2 \pi \alpha^{\prime}}\right) .
$$

The important point is that the potential $V_{A}(\alpha)$ is periodic under $\alpha \rightarrow \alpha+2 \pi / N_{c}$ due to the gauge symmetry of the boundary supersymmetric Yang-Mills (SYM) theory [7]. ${ }^{2}$ As a result, the total effective potential, the sum of $V_{A}(\alpha)$ and the probe action, is also periodic under $\mu_{I} / T \rightarrow \mu / T+2 \pi / N_{c}$, since a finite shift of $\mu_{I} / T$ can be absorbed into $\alpha$ as understood from Eq. (1.2). The role of $\mu_{I}$ in the probe action is to control the minimum of the \footnotetext{
here.

${ }^{1}$ The disc $D_{2}$ is a part of the black hole geometry considered ${ }^{2}$ See also Appendix A.
} 
effective total potential of $\alpha$ as seen in the RW phase transition $[7,8]$.

The purpose of this paper is to extend the analysis performed for $\mu_{I}$ in the top-down models to a bottom-up model which has been used to study the color superconductivity in QCD [14-16]. Through the extension, we could get phase diagrams for our model in the region of $\mu_{I}$ with RW transitions. And an implication of our holographic model is discussed. Especially, when the backreaction of flavor fermions is included, we find a $\mu_{I}$-dependent critical curve of confinement/deconfinement transition. In this case, we could show the usefulness of a simple continuation, $\mu \rightarrow i \mu_{I}$, in terms of the critical curve obtained for real $\mu$. This usefulness is supported by the fact that we can set $\alpha=0$ near $\mu_{I}=0$.

In the next section, the extended bottom-up model is proposed and the actions are estimated for confinement and deconfinement phases. In Sec. III, the RW transitions are investigated in the probe approximation, and for the backreacted case. Then the phase diagrams are given. In Sec. IV, the validity of the continuation near $\mu=0$ is discussed by comparing the critical curve near $\mu=0$ for the holographic model and for the dual QCD theories. A problem related to a wide periodicity of the potential of $\mu_{I}$ is discussed in Sec. V. Our summary is given in the final section.

\section{A BOTTOM-UP MODEL}

A bottom-up model, which was used before to study the superconductivity of QCD [14], is given in a slightly modified form of the following action for the Euclidean space-time to investigate QCD with the imaginary chemical potential. The model consists of two parts:

$$
S=S_{\mathrm{bu}}+S_{F_{(4)}} .
$$

The first term is given as

$$
\begin{gathered}
S_{\text {bu }}=\int d^{6} x \sqrt{-g}\left(\mathcal{L}_{\text {Gravity }}+\mathcal{L}_{\mathrm{CSC}}\right) \\
\mathcal{L}_{\text {Gravity }}=\frac{1}{2 \kappa_{6}^{2}}\left(\mathcal{R}+\frac{20}{L^{2}}\right) \\
\tilde{\mathcal{L}}_{\mathrm{CSC}}=-\frac{1}{4} \tilde{F}^{2}-\left|D_{\mu} \psi\right|^{2}-m^{2}|\psi|^{2} \\
\tilde{F}_{\mu \nu}=\partial_{\mu} A_{\nu}-\partial_{\nu} A_{\mu}+\frac{B_{\mu \nu}}{2 \pi \alpha^{\prime}}, \quad D_{\mu} \psi=\left(\partial_{\mu}-i q A_{\mu}\right) \psi
\end{gathered}
$$

This action $S_{\mathrm{bu}}$ is proposed as a gravitational model dual to the SYM theory, which could include the strongly interacting flavor fermions with the chemical potential $\mu$, when the space-time is Lorentzian where the field $B_{\mu \nu}$ is neglected. On the other hand, for the Euclidean space-time, the field $B_{\mu \nu}$ must be retained, since the phase of the Polyakov loop plays the main role in the RW transitions. In the gravitational part $\mathcal{L}_{\text {Gravity }}$, which is dual to the pure SYM theory, the scale $L$ denotes the anti-de Sitter (AdS) radius. The part given by $\tilde{\mathcal{L}}_{\mathrm{CSC}}$ is dual to the flavor fermion system. In the case of the top-down models, it is usually given by the D-branes. In the present bottom-up model, however, only a part of the D-branes appears in the bulk, and the D-brane profiles are obscure. The study of how this model can be lifted up to some appropriate top-down holographic theory is postponed.

In the present case, the theory is set in the Euclidean space-time. It is obtained from the Lorentzian action by the Wick rotation of both the time and fields. We notice here that the Kalb-Ramond field is added through $\tilde{F}_{\mu \nu}$ as given in Eq. (2.5). This form of $\tilde{F}_{\mu \nu}$ is implied from the D-brane action. And $\psi$ denotes a charged scalar, which is supposed to be dual to the Cooper pair of the color charged fermions. Its baryon number charge is assigned as $q$. We could show, by supposing an appropriate conformal dimension and the mass $m^{2}$, that there is no nontrivial solution for $\psi$ in the region of small and negative $\mu^{2}$ [14]. ${ }^{3}$ Since $\mu^{2}$ is negative for imaginary $\mu$, we can neglect $\psi$ hereafter because we are considering the case with imaginary chemical potential.

Then, the system can be solved by setting $\tilde{A}_{0}=\tilde{\phi}$, where $\tilde{A}_{\mu}$ is defined by

$$
\tilde{F}_{\mu \nu} \equiv \partial_{\mu} \tilde{A}_{\nu}-\partial_{\nu} \tilde{A}_{\mu}
$$

This replacement can be justified, since $B$ is introduced with $d B=0$. In this case, we will find the same form for the equations of motion as that of the real $\mu$ theory given in the Lorentzian space-time. However, in the present case, we must notice that the solution $\tilde{\phi}$ is not simply a chemical potential, but a combination of the chemical potential and $\alpha$, as found from Eq. (1.2). This fact implies that we can obtain the solutions with $\tilde{\phi}$ for $\mu_{I}$ from that of the real $\mu$ by a replacement, $\mu / T \rightarrow i\left(\mu_{I} / T-\alpha\right)$.

As for the action $S_{F_{(4)}}$, this is necessary to study the potential of $\alpha$. Its explicit form and an effective potential of the $B$ field are given in Sec. II B.

\section{A. Bulk solutions}

As mentioned above, neglecting $\tilde{\phi}$, we can obtain solutions with the imaginary chemical potential $\mu_{I}$. We give three solutions dual to the ground states of the pure YM fields, and they are compared. Two of them are the solutions of $L_{\text {Gravity }}$ only, and thus they are independent

\footnotetext{
${ }^{3}$ On this point, we can see the details in Ref. [14]. A brief explanation is given in Appendix B.
} 
of $\mu$. The third solution is constructed by considering the backreaction from $\tilde{F}^{2}$.

(1) AdS soliton solution: This represents the lowtemperature confinement phase, and it is given as

$$
d s^{2}=r^{2}\left(\delta_{\mu \nu} d x^{\mu} d x^{\nu}+f(r) d w^{2}\right)+\frac{d r^{2}}{r^{2} f(r)},
$$

where

$$
f(r)=1-\left(\frac{r_{0}}{r}\right)^{5}, \quad r_{0}=\frac{2}{5 R_{w}},
$$

and $2 \pi R_{w}$ denotes the compactified length of $w$.

(2) AdS-Schwarzschild solution: This solution corresponds to the high-temperature deconfinement phase,

$$
d s^{2}=r^{2}\left(f d t^{2}+\Sigma_{i}^{3}\left(d x^{i}\right)^{2}+d w^{2}\right)+\frac{d r^{2}}{r^{2} f(r)},
$$

where

$$
f(r)=1-\left(\frac{r_{0}}{r}\right)^{5}, \quad r_{0}=\frac{2}{5 R_{w}} .
$$

(3) Reissner-Nordstrom (RN) solution: In this case, the backreaction of flavor is taken into account. It represents the high-temperature deconfinement phase.

The background of $\mathrm{RN}$ is given as the solution of the following action:

$$
S_{G}=\int d^{6} x \sqrt{-g}\left\{\frac{1}{2 \kappa_{6}^{2}}\left(\mathcal{R}+\frac{20}{L^{2}}\right)-\frac{1}{4} \tilde{F}^{2}\right\}
$$

which includes the flavor part. We get the following RN solution:

$$
\begin{gathered}
d s^{2}=r^{2}\left(g d t^{2}+\Sigma_{i}^{3}\left(d x^{i}\right)^{2}+d w^{2}\right)+\frac{d r^{2}}{r^{2} g(r)}, \\
g=1-\left(1-\frac{3 \tilde{\mu}^{2}}{8 r_{+}^{2}}\right)\left(\frac{r_{+}}{r}\right)^{5}-\frac{3 \tilde{\mu}^{2} r_{+}^{6}}{8 r^{8}}, \\
\tilde{A}_{0}=\tilde{\phi}=\tilde{\mu}\left(1-\frac{r_{+}^{3}}{r^{3}}\right) .
\end{gathered}
$$

Here $r_{+}$denotes the horizon of the charged black hole, and the temperature is given as

$$
T=\frac{1}{4 \pi}\left(5 r_{+}+\frac{9 \tilde{\mu}^{2}}{8 r_{+}}\right) .
$$

Here, $\tilde{\mu}$ is defined by

$$
-\frac{\tilde{\mu}}{T}=\int_{D_{2}} \tilde{F}=\int_{D_{2}}\left(F+\frac{B}{2 \pi \alpha^{\prime}}\right)=\alpha-\frac{\mu_{I}}{T} .
$$

The action densities for these solutions-(1) AdS-Soliton, (2) AdS-Schwarzschild, and (3) $\mathrm{RN}$ - are given as

$$
\begin{gathered}
S_{1} / V_{3}=-r_{0}^{5} v_{2}=-r_{0}^{5} \frac{4 \pi}{5 r_{0}} \frac{1}{T}, \\
S_{2} / V_{3}=-r_{0}^{5} v_{2}=-r_{0}^{5}\left(\frac{4 \pi}{5 r_{0}}\right)^{2},
\end{gathered}
$$

$S_{3} / V_{3}=-r_{+}^{5}\left(1-\frac{3 \tilde{\mu}^{2}}{8 r_{+}^{2}}\right) v_{2}=-r_{+}^{5}\left(1-\frac{3 \tilde{\mu}^{2}}{8 r_{+}^{2}}\right) \frac{4 \pi}{5 r_{0}} \frac{1}{T}$,

where $v_{2}=\int_{0}^{\beta} d \tau \int d w$ and $V_{3}=\int d x d y d z$. In the $\mu_{I}-T$ plane, we can find the phase diagram by comparing the above three actions. We find that the phase of solution (2) is not realized when solution (3) is added.

\section{B. Potential of Kalb-Ramond field}

Consider the Kalb-Ramond field $B$, which is introduced in terms of $\alpha$, which is defined by Eq. (1.1). The present bottom-up model would be related to the D4/D8 model of type-IIA string. Then the bulk action, which could provide the potential of $\alpha$, might be given as

$S_{F_{(4)}}=-\frac{1}{2 \kappa_{6}^{2}}\left(\int d^{6} x \sqrt{g} \frac{1}{12} F_{(4)}^{2}-\int B \wedge F_{(4)}\right)$.

In the RN background, Eqs. (2.12)-(2.15), this action is estimated for a constant field $F_{123 w}$ and $\alpha$. We obtain

$$
S_{F_{(4)}}=-\frac{V_{4}}{2 \kappa_{6}^{2}}\left(\frac{\beta}{6 r_{+}^{3}} F_{123 w}^{2}-\alpha F_{123 w}\right),
$$

where $V_{4}=\int d^{3} x d w=\beta V_{3}, V_{3}=\int d x^{3}$, and $r_{+}$is used as the lower limit of the integration of $r$ as $\int_{r_{+}}^{\infty} d r$. It should be replaced by $r_{0}$ in the case of solutions (1) and (2). Solving the equation of motion of $F_{123 w}$, we find the solution as $F_{123 w}=3 r_{+}^{3} \alpha / \beta$. Then the potential is obtained as 


$$
S_{F_{(4)}} / V_{3} \equiv V_{A}=\frac{1}{2 \kappa_{6}^{2}} \frac{3 r_{+}^{3}}{2} \alpha^{2}
$$

Due to the gauge symmetry of the dual SYM theory, the above result should be written as

$$
V_{A}=\min _{n \in \mathbb{Z}} \frac{1}{2 \kappa_{6}^{2}} \frac{3 r_{+}^{3}}{2}\left(\alpha-\frac{2 \pi n}{N_{c}}\right)^{2} .
$$

\section{ROBERGE-WEISS TRANSITIONS}

\section{A. Probe approximation}

In the probe approximation, the gauge term $\tilde{L}_{\mathrm{CSC}}$ is treated as the probe for the background given by the gravitational part. In this case, the background actions are given by $S_{1}$ and $S_{2}$, and we find the critical line of confinement/deconfinement by comparing the two bulk actions. Then, the critical line is found as

$$
T=\frac{5 r_{0}}{4 \pi} .
$$

This is independent of $\mu_{I}$; therefore, the critical line is common to the case of real $\mu$. The probe part defined by Eq. (2.4) is solved under these backgrounds. The equations of motion of $\tilde{\phi}$ are given by the ansatz $\tilde{A}=\tilde{A}_{\mu} d x^{\mu}=\tilde{\phi}(r) d t$.

In the confinement phase, the background is given by solution (1), and the equation for $\tilde{\phi}$ is given as

$$
\tilde{\phi}^{\prime \prime}+\left(\frac{4}{r}+\frac{f^{\prime}}{f}\right) \tilde{\phi}^{\prime}=0
$$

We find that the allowed solution of this equation is $\tilde{\phi}=$ const. This solution gives no contribution to the free energy. On the other hand, the Kalb-Ramond field has no meaning in the confinement phase. So there is no new phase transition in this phase.

An interesting phenomenon is observed in the deconfinement phase with the background of solution (2). In this case, we have the equation

$$
\tilde{\phi}^{\prime \prime}+\frac{4}{r} \tilde{\phi}^{\prime}=0 .
$$

This equation is solved as

$$
\tilde{\phi}=\tilde{\mu}\left(1-\frac{r_{0}^{3}}{r^{3}}\right)
$$

This solution provides a nontrivial contribution to the free energy as shown below. The probe action is given as

$$
\begin{aligned}
S_{\mathrm{CS}}^{E} & =-\int d x^{6} \sqrt{-g}\left(-\frac{1}{4} \tilde{F}^{2}\right) \\
& =\int d x^{5} \int_{r_{0}}^{\infty} d r \frac{r^{4}}{2}\left(\tilde{\phi}^{\prime}\right)^{2} \\
& =\int d x^{3} V_{f},
\end{aligned}
$$

where

$$
S_{\mathrm{CS}}^{\mathrm{E}} / V_{3}=V_{f}=\frac{3}{2}\left(\frac{4 \pi}{5}\right)^{3} T \tilde{\mu}^{2} .
$$

Then $\tilde{\mu}$ is replaced by $\mu_{I}$ and $\alpha$ by Eq. (2.16), and we obtain

$$
V_{f}=\frac{3}{2}\left(\frac{4 \pi T}{5}\right)^{3}\left(\alpha-\mu_{I} / T\right)^{2} .
$$

Now, from this $V_{f}$ and Eq. (2.23), where $r_{+}$is replaced by $r_{0}(=4 \pi T / 5)$, we find

$$
\begin{aligned}
V_{\mathrm{eff}}= & V_{A}+V_{f}(\alpha) \\
= & \min _{n \in \mathbb{Z}} \frac{1}{2 \kappa_{6}^{2}} \frac{3}{2}\left(\frac{4 \pi T}{5}\right)^{3}\left(\alpha-\frac{2 \pi n}{N_{c}}\right)^{2} \\
& +\frac{3}{2}\left(\frac{4 \pi T}{5}\right)^{3}\left(\alpha-\mu_{I} / T\right)^{2} .
\end{aligned}
$$

From this effective potential, we can see the RobergeWeiss transition for the state defined by the value of $\alpha$. An example of this transition is read from Fig. 1, in which we can see the transition from $\langle\alpha\rangle=0$ to the $\langle\alpha\rangle=2 \pi / 3$ vacuum state-namely, from the phase (b0) to (b1) in Fig. 2. The resultant phase diagram obtained from the above $V_{\text {eff }}$ is shown in Fig. 2. Here, the relative ratio of the probe term and the $B$ term $V_{A}$ is set by the relation $1 / 2 \kappa_{6}^{2}=10$ for simplicity.

Finally, we give an effective potential under the quenched approximation of the gauge field configurations which provide the real Polyakov loop. This potential is obtained from Eq. (3.10) by considering the functions at $\alpha=2 \pi n$ where $n \in \mathbb{Z}$, and it is found by picking up the minimum parts,

$$
V_{\mathrm{eff}}^{(0)}=\min _{n \in \mathbb{Z}} \frac{3}{2}\left(\frac{4 \pi T}{5}\right)^{3}\left(2 \pi n-\mu_{I} / T\right)^{2} .
$$

This potential has the period $2 \pi$ with respect to $\mu_{I} / T$ as expected, and it is shown in Fig. 3. This period can be understood from the phase of the boundary condition imposed on the fundamental fermions of the theory. In the present article, this potential is not used; however, this periodicity is seen, for example, in the calculation of the chiral condensate in the gauge configurations of the real 


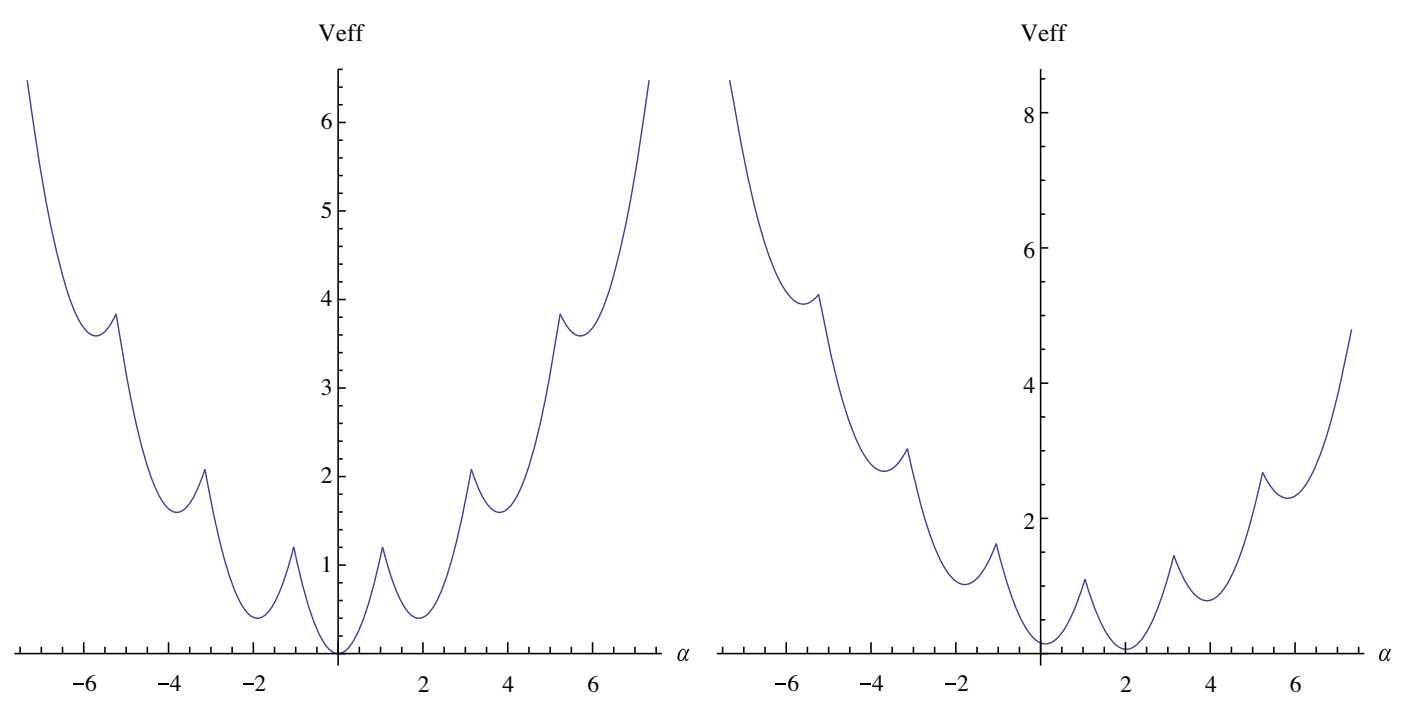

FIG. 1. $V_{\text {eff }}$ for $\mu_{I} / T=0$ (left) and $\mu_{I} / T=0.6 a$ (right).

Polyakov loops $[5,6]$. This point is given more discussion in Sec. V.

\section{B. Backreacted case}

When the backreaction of the flavor part is taken into account, the deconfinement background is replaced by the RN solution (3), since $S_{3}<S_{2}$. The action $S_{3}$ is given in Eq. (2.19). We notice that the $\tilde{\mu}$ dependence of $S_{3}$ also comes from $r_{+}$. Actually, by using Eq. (2.15), $r_{+}$is written as

$$
r_{+}=\frac{2 \pi T}{5}\left(1+\sqrt{1-\frac{45 \tilde{\mu}^{2}}{8} /(2 \pi T)^{2}}\right) .
$$

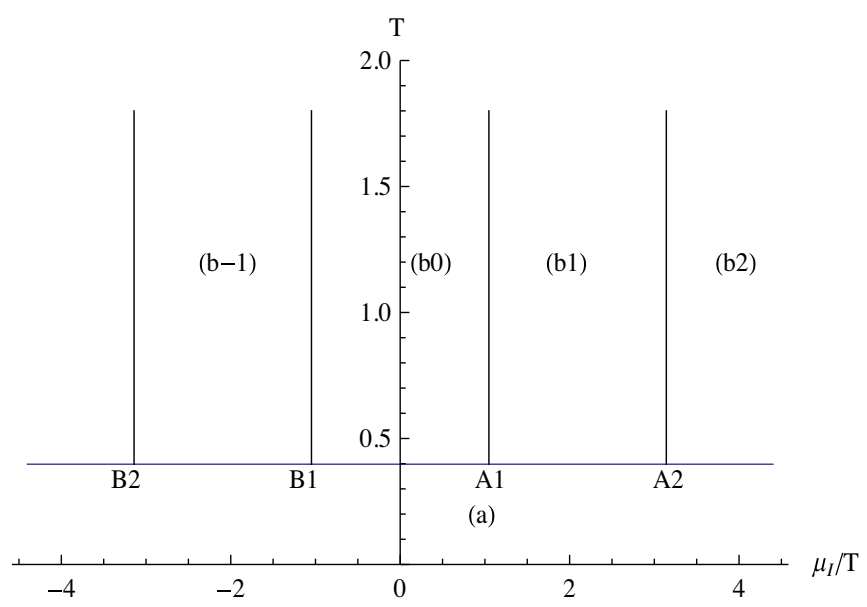

FIG. 2. Phase diagram for probe approximation. The horizontal critical line separates the confinement and deconfinement phases. In the large- $T$ deconfinement phase, the RW transitions are shown by the vertical critical lines. The points $A_{1} \sim B_{2}$ represent tricritical points.
This solution is useful for $|\tilde{\mu}| / T<\sqrt{32 \pi^{2} / 45}(=2.65)$ due to the reality of $r_{+}$. For $|\tilde{\mu}| / T>\sqrt{32 \pi^{2} / 45}$, the system becomes unstable and decays to the stable confinement phase expressed by solution (1), the AdS-soliton background.

To observe the RW transitions, it is helpful to see the phase diagram for $\alpha=0$-namely, for $\tilde{\mu}=\mu_{I}$. This diagram is obtained by comparing $S_{3}$ with $S_{1}$ for $\alpha=0$, and it is shown in Fig. 4. The unstable region of solution (3) mentioned above is shown by region (B) in this figure for $\alpha=0$, and the regions (A) for the Reissner-Nordstrom deconfinement phase and (C) for the AdS soliton confinement phase are also shown. Since region (C) must be replaced by the confinement phase of (B), the deconfinement region (A) is restricted to the definite region of $\left|\mu_{I}\right| / T$, $\left|\mu_{I}\right| / T \leq \sqrt{32 \pi^{2} / 45}$, as mentioned above. This restriction comes from the backreaction.

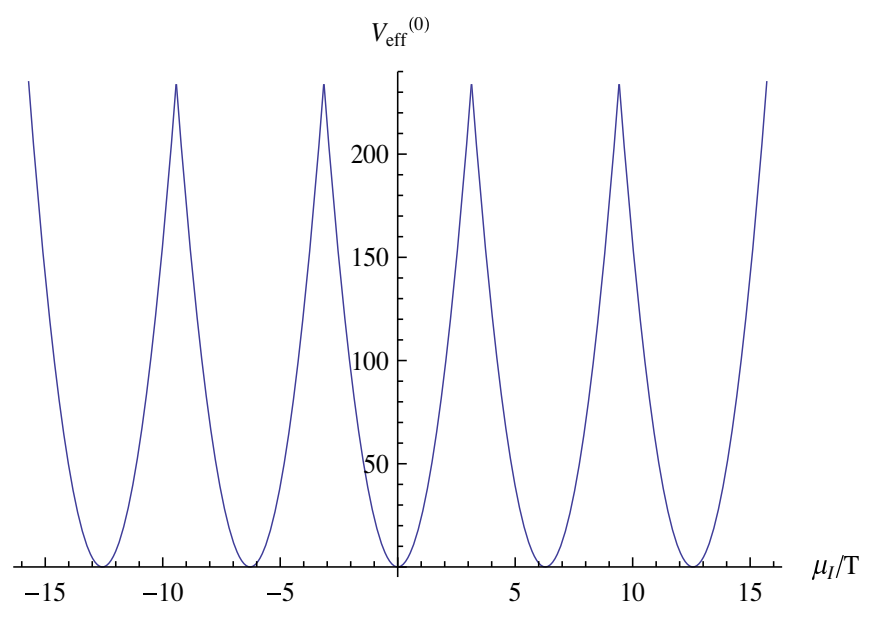

FIG. 3. $\quad V_{\mathrm{eff}}^{(0)}$. 


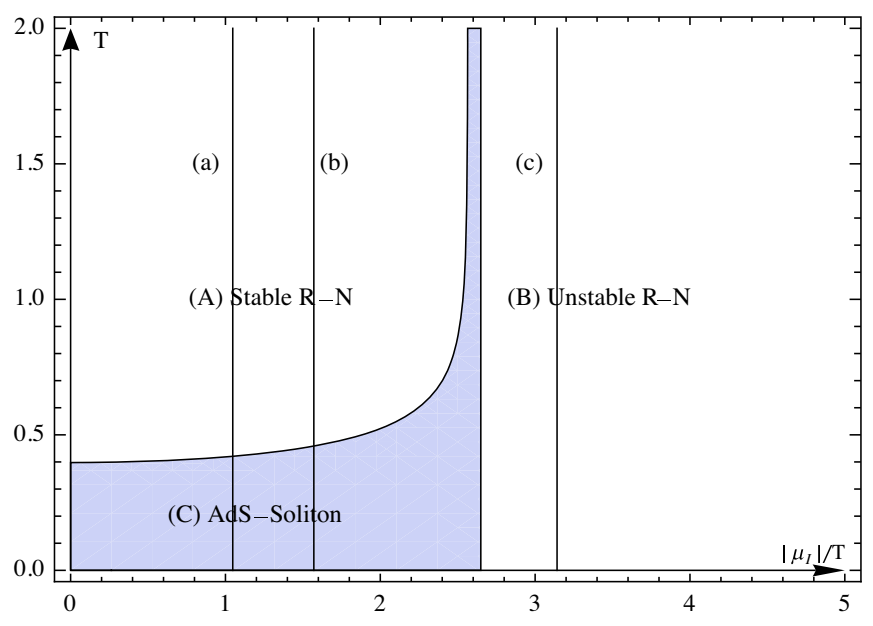

FIG. 4. Phase diagram of the backreacted case for $r_{0}=1$ and $\alpha=0$. The lines (a), (b), and (c) represent $\left|\mu_{I}\right| / T=\pi / 3, \pi / 2$, and $\pi$, respectively.

Now we study the RW transitions in the deconfinement phase of region (A) by reviving $\alpha$. In the present case, the effective potential is given as

$$
V_{\mathrm{eff}}=V_{A}+V_{f}^{\mathrm{RN}}=\min _{n \in \mathbb{Z}} \frac{1}{2 \kappa_{6}^{2}} \frac{3 r_{+}^{3}}{2}\left(\alpha-\frac{2 \pi n}{N_{c}}\right)^{2}+V_{f}^{\mathrm{RN}} .
$$

The first term, $V_{A}$, is obtained in Eq. (2.23) for the background of $\mathrm{RN}$, and the second term is given as

$$
S^{\mathrm{RN}}=\frac{1}{2 \kappa_{6}^{2}} \int d^{6} x \sqrt{-g}\left\{\mathcal{R}+\frac{20}{L^{2}}-\frac{1}{4} \tilde{F}^{2}\right\}=\int d x^{3} V_{f}^{\mathrm{RN}} .
$$

Using Eq. (2.19), we find

$$
V_{f}^{\mathrm{RN}}=-\frac{1}{2 \kappa_{6}^{2}} r_{+}^{5}\left(1-\frac{3 \tilde{\mu}^{2}}{8 r_{+}^{2}}\right) \frac{4 \pi}{5 r_{0}} \frac{1}{T} .
$$

This potential is the part dual to the combined system of SYM fields and flavor fermions with an imaginary chemical potential $\mu_{I}$.

Here, we notice that $V_{A}$ also depends on $\tilde{\mu}$ through $r_{+}$. This fact can be interpreted as a kind of backreaction to the Kalb-Ramond potential from the flavor fermions. In order to understand this backreaction, we restrict the region of $\left|\mu_{I}\right|$ to the region of small $\left|\mu_{I} / T-\alpha\right|$. Then we can expand $V_{\text {eff }}$ in the series of $-\left(\mu_{I} / T-\alpha\right)^{2}$. The expanded potential is retained up to the order of $\left|\mu_{I}-\alpha / \beta\right|^{2}$, and we obtain

$V_{A}=\min _{n \in \mathbb{Z}} \frac{1}{2 \kappa_{6}^{2}}\left(\frac{64 \pi^{3}}{125}-\frac{27 \pi}{50}\left(\alpha-\frac{\mu_{I}}{T}\right)^{2}\right) T^{3}\left(\alpha-\frac{2 \pi n}{N_{c}}\right)^{2}+\cdots$,

$$
V_{f}^{\mathrm{RN}}=\frac{1}{2 \kappa_{6}^{2}}\left(-\frac{1024 \pi^{5}}{3124}+\frac{96 \pi^{3}}{125}\left(\alpha-\frac{\mu_{I}}{T}\right)^{2}\right) T^{3}+\cdots .
$$

We find that this result is almost equal to the probe approximation, except that the coefficient of $V_{A}$ is slightly modified. In fact, we can see similar behavior of the potential to that of the probe approximation. Then we could find the expected RW transitions in this case also. Furthermore, the qualitative behaviors of the potential are maintained even if the full form of the potential is used. So, we show here the RW transitions in terms of the full form of the potential.

In Fig. 5, the effective potential with two values of $\mu_{I} / T$ is shown in order to display the RW transition from $\langle\alpha\rangle=0$ to $\langle\alpha\rangle=a$ with the period $a$, which should be set as $2 \pi / N_{c}$. It is easy to find other periodic transitions. The resultant phase diagram with the RW transitions is shown in Fig. 6.

Here, we should notice that the periodicity of $\mu_{I} / T$ in $V_{\text {eff }}$ implies $\left|\mu_{I} / T\right|<\pi / N_{c}$. This and the constraint $\left|\mu_{I}\right| / T \leq \sqrt{32 \pi^{2} / 45}$ given above leads to the constraint

$$
N_{c}>1.18 \text {. }
$$

In spite of the fact that $N_{c}$ must be a large integer to justify the holographic approach, we are allowed to use the holography up to $N_{c}=2$ at this stage.

\section{COMPARISON WITH QCD NEAR $\mu=0$}

As shown above, the $\mu$-dependent critical line obtained for real $\mu$ can be continued to the imaginary $\mu$ region and used there. The form of the critical line near $\mu=0$ is given as [17]

$$
\frac{T}{T_{0}}=1-a\left(\frac{\mu}{T_{0}}\right)^{2}+\cdots
$$

where $T_{0}$ denotes the critical temperature at $\mu=0$, and $a$ is a dimensionless constant, which depends on the parameters of the theory. It has been obtained also in the lattice QCD for $\mu^{2}<0$ without bothering with the sign problem. Then it is meaningful to compare the result obtained in lattice QCD with the holographic result given here.

For the probe approximation, we find $a=0$, since the critical line is independent of $\mu$. So, we consider the RN background case, where the $\mu$-dependent critical curve is obtained from the equation $S_{1}=S_{3}$. And we find

$$
a=\frac{15}{32 \pi^{2}}=0.0475 \text {. }
$$



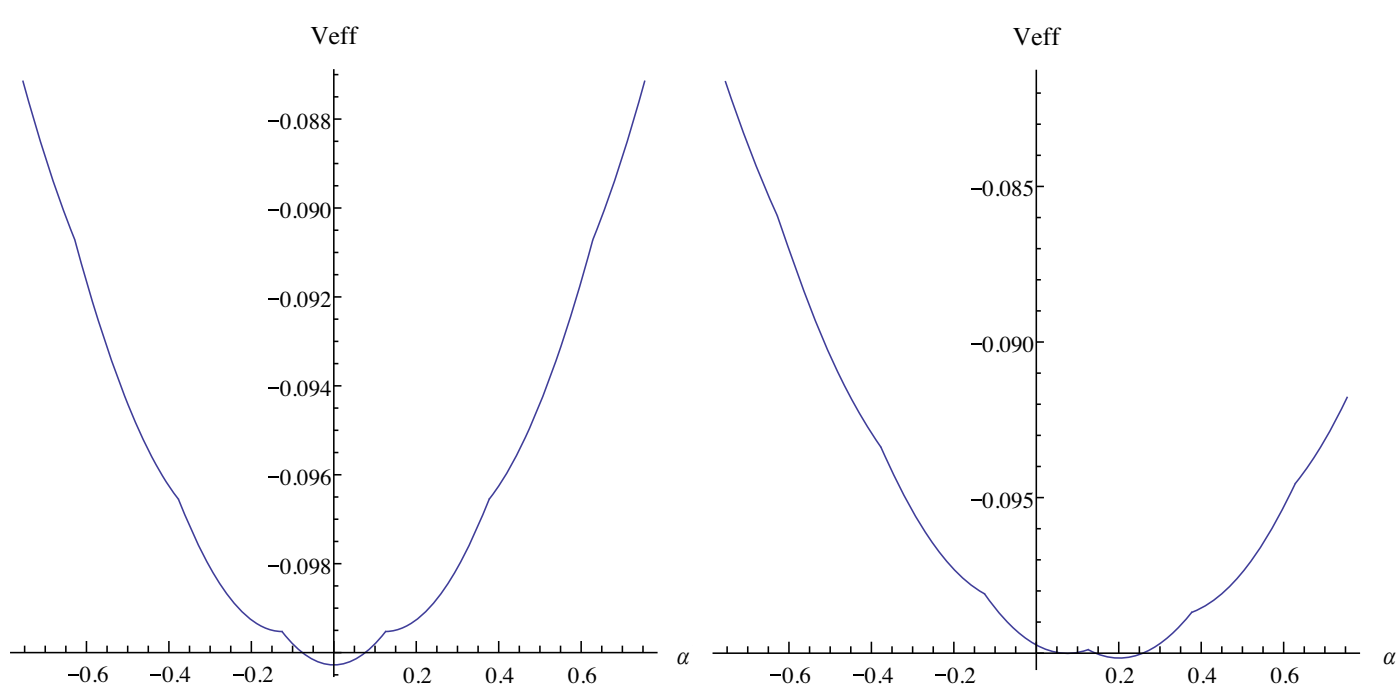

FIG. 5. Full form of $V_{\text {eff }}$ for the RN backreacted case. The left plot shows $\mu_{I} / T=0$, and the right displays $\mu_{I} / T=0.6 a$ with the period of $a=2 \pi / 25$.

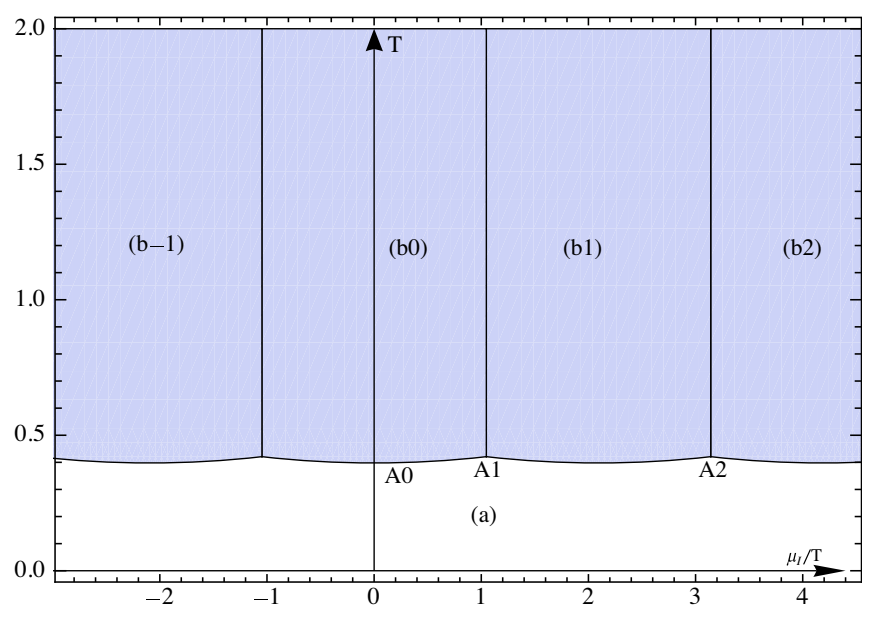

FIG. 6. Phase diagram for RW transitions under the backreacted background solution for $N_{c}=3$. In the RN deconfinement phase, the phases are separated by the vertical critical lines into the regions $(\mathrm{b}-1) \sim(\mathrm{b} 2)$ for $\langle\alpha\rangle=-2 \pi / 3,0,2 \pi / 3,4 \pi / 3$. The area (a) corresponds to the AdS soliton confinement phase. The points $A_{1}$ and $A_{2}$ represent the tricritical points.

In the lattice QCD, the coefficient is obtained by the form

$$
a=\kappa N_{c}^{2} \text {. }
$$

For confinement transition, we find many simulation results. We pick up several examples, to compare with the holographic result, however, where $N_{c}$ is assumed to be large. The examples of estimations of $\kappa$ from lattice QCD data in the $2+1$ and $2+1+1$ flavor systems are $0.0066 \pm 20$ [18], $0.013 \pm 0.003$ [19], $0.0135 \pm 0.002$ [20], $0.0149 \pm 0.0021$ [21], and $0.020 \pm 0.004$ [22]. Comparing this with Eq. (4.2) and using Eq. (4.3), we find $N_{c}=1.76$ for $\kappa \sim 0.0153$. This is consistent with the result $N_{c} \geq 1.2$ obtained in our backreacted case.

In addition, there is the estimation of $\kappa$ by using the Polyakov loop extended Nambu-Jona-Lasinio (NJL) model with the mean-field approximation as $\kappa_{\chi}=0.017 \pm$ 0.001 [23]; it should be noted that this value is estimated from the isospin chemical potential, but $\kappa$ should be exactly same in both cases, at least in the mean-field approximation. In the case of the PNJL model, there is the estimation of $\kappa$ for the confinement-deconfinement crossover line as $0.004 \pm 0.001$ and $0.003 \pm 0.001$; the former one is evaluated from the Polyakov loop, and the latter one uses the quark number holonomy [24].

\section{MORE ABOUT THE PERIODICITY}

In the previous section, we discussed possible connections between lattice QCD simulations near $\mu^{2}=0$. In this section, we discuss deeper properties of the periodicity appearing in QCD. In full QCD, we should have the RW periodicity as explained and demonstrated above. It is, however, well known that we should have the $2 \pi$ periodicity for $\mu_{I} / T=\theta$ instead of the Roberge-Weiss (RW) periodicity in the lattice QCD simulation when we fix gauge configurations at $\mu=0$ or the pure gauge limit where dynamical quarks are not taken into account; the latter one corresponds to the quenched limit. In this case, any quantities such as the pressure, the entropy density, and the cumulant lose the RW periodicity, and thus the minimal period becomes $2 \pi$ because the grand canonical partition function does not have the RW periodicity. It should be noted that we need the Polyakov loop phase flip to consider the RW periodicity in the limits as discussed in Refs. [25,26].

The $2 \pi$ periodicity has been used in the calculation of the dual quark condensation. The actual definition of the dual quark condensation is given by 


$$
\Sigma^{(n)}=\int_{0}^{2 \pi} \frac{d \phi}{2 \pi} \sigma(\phi) e^{i n \theta} d \phi,
$$

where $\sigma(\phi)$ is the chiral condensate with the phase of the boundary condition, $0 \leq \phi \leq 2 \pi$, which is related with the dimensionless imaginary chemical potential as

$$
\phi=\theta+\pi
$$

In the heavy quark mass regime, it has a clear relation with the Polyakov loop by using the Dirac mode expansion $[5,6]$. Since the dual quark condensate is calculated from the chiral condensate, this quantity may bridge the chiral and the Polyakov loop dynamics in QCD. Details of the dual quark condensate have been discussed in the lattice QCD simulation [5,6], the Dyson-Schwinger equation [27], and QCD effective models [28-30]. In principle, we can investigate the Polyakov loop behavior at $\mu=0$ from the $\phi$-dependent chiral condensate even if calculation of the Polyakov loop is difficult or impossible.

In the strict probe limit of the holographic model, we also face the same situation of the lattice QCD simulation in the quenched limit: we only have the $2 \pi$ trivial periodicity. For consistency between the holographic model and the lattice QCD simulation in both limits, we should reproduce the $2 \pi$ periodicity in addition to the RW periodicity. Also, it is good to calculate the dual quark condensate in the holographic model to discuss the relation between the chiral condensate and the Polyakov loop. In addition, if we can understand how to control the boundary condition in the holographic model, we will contact with the $\mathcal{Z}_{\mathrm{N}_{c}}$ twisted QCD, an interesting QCD-like theory in the viewpoint of the sign problem appearing in the lattice simulation. One possibility for introducing the $2 \pi$ trivial periodicity in the probe limit is imposing a $2 \pi$ periodic form of $\theta$ in the mapping of $\mu$ from $A_{0}$ : we need special care for the $2 \pi$ periodicity issue of the dimensionless imaginary chemical potential.

\section{SUMMARY}

We have studied here the phase structure and phase transition behaviors ranging from real to imaginary chemical potential, using a bottom-up holographic model that was introduced to investigate color superconductivity in QCD. From the general framework of QCD, one knows that the QCD partition function possesses a certain periodicity, the Roberge-Weiss (RW) periodicity, at the imaginary chemical potential region. Our interest was to see how the analytic continuation of the chemical potential works. To this end, we have computed the effective potential of the model by including the Kalb-Ramond field in the bulk. Unlike the previous studies based on a top-down approach, there is an advantage of our bottom-up approach in that one can evaluate the effect of backreaction. As a result, we have observed the RW periodicity as well as the $2 \pi$ periodicity appropriately. We have further investigated the behavior of the critical line near $\mu=0$ and tried to see the validity of our analysis. Our results have been compared with those obtained from lattice QCD and effective models such as the Polyakov loop extended NJL model.

\section{ACKNOWLEDGMENTS}

The authors would like to thank Takeshi Morita for the helpful discussion. K. K. is supported by Grants-in-Aid for Scientific Research from JSPS (No. 18K03618).

\section{APPENDIX A: ROBERGE-WEISS PERIODICITY IN THE OPERATOR FORMALISM}

Since the Roberge-Weiss periodicity must be independent of the gauge fixing condition except for the global topology of gauge configuration, the mechanism of the periodicity seems to result strongly from the quark side. In this appendix, we present a brief demonstration of the mechanism in the operator formalism. In the path-integral representation, the periodicity is related to the boundary condition along imaginary time. Therefore, we omit the spatial degrees of freedom in the following discussions.

Let us begin with a single-fermion system. The coherent states are defined by

$$
\begin{aligned}
|\xi\rangle & =\left(1-\xi a^{\dagger}\right)|0\rangle=|0\rangle-\xi|1\rangle, \\
\langle\xi| & =\frac{1}{i}\langle 0|(\xi-a)=\frac{1}{i}(\langle 0| \xi-\langle 1|) .
\end{aligned}
$$

Using the integration rule $\int d \xi \xi=i$, the trace of an operator $\mathcal{O}$ is calculated as

$$
\int d \xi\langle\xi|\mathcal{O}|-\xi\rangle=\langle 0|\mathcal{O}| 0\rangle+\langle 1|\mathcal{O}| 1\rangle=\operatorname{tr} \mathcal{O} .
$$

This leads to the antiperiodic boundary condition in a pathintegral representation [31].

In a particle-antiparticle system, the coherent states which satisfy

$$
\begin{aligned}
a|\xi, \bar{\xi}\rangle & =\xi|\xi, \bar{\xi}\rangle, & b|\xi, \bar{\xi}\rangle & =\bar{\xi}|\xi, \bar{\xi}\rangle \\
\langle\xi, \bar{\xi}| a & =\langle\xi, \bar{\xi}| \xi, & \langle\xi, \bar{\xi}| b & =\langle\xi, \bar{\xi}| \bar{\xi}
\end{aligned}
$$

are constructed as follows:

$$
\begin{aligned}
|\xi, \bar{\xi}\rangle & =\left(1-\xi a^{\dagger}\right)\left(1-\bar{\xi} b^{\dagger}\right)|0,0\rangle \\
& =|0,0\rangle-\xi|1,0\rangle-\bar{\xi}|0,1\rangle+\bar{\xi} \xi|1,1\rangle, \\
\langle\xi, \bar{\xi}|=\frac{1}{i^{2}}\langle 0,0|(\bar{\xi}-b)(\xi-a) & \\
= & \frac{1}{i^{2}}(\langle 0,0| \bar{\xi} \xi+\langle 1,0| \bar{\xi}-\langle 0,1| \xi+\langle 1,1|) .
\end{aligned}
$$


Corresponding to boundary conditions as $\psi(\beta)=-\sigma \psi(0)$ and $\bar{\psi}(\beta)=-\sigma^{*} \bar{\psi}(0)$ in the path-integral representation, the calculation of the trace is modified as

$$
\begin{aligned}
\operatorname{tr}_{\sigma} \mathcal{O}= & \int d \xi d \bar{\xi}\left\langle\xi, \bar{\xi}|\mathcal{O}|-\sigma \xi,-\sigma^{*} \bar{\xi}\right\rangle \\
= & \langle 0,0|\mathcal{O}| 0,0\rangle+\sigma\langle 1,0|\mathcal{O}| 1,0\rangle \\
& +\sigma^{*}\langle 0,1|\mathcal{O}| 0,1\rangle+\langle 1,1|\mathcal{O}| 1,1\rangle .
\end{aligned}
$$

(This reduces to the normal trace in the case of $\sigma=1$.)

Note that

$$
\left|-\sigma \xi,-\sigma^{*} \bar{\xi}\right\rangle=\mathrm{e}^{i \gamma\left(a^{\dagger} a-b^{\dagger} b\right)}|-\xi,-\bar{\xi}\rangle \quad\left(\sigma=\mathrm{e}^{i \gamma}\right),
$$

and then we obtain

$$
\operatorname{tr}_{\sigma} \mathcal{O}=\operatorname{tr}\left(\mathcal{O} \mathrm{e}^{i \gamma\left(a^{\dagger} a-b^{\dagger} b\right)}\right) .
$$

Now, the grand potential $Z(\beta, \mu)$ of the $S U(N)$ local gauge theory is invariant under the $Z_{N}$ transformation associated with the $Z_{N}$-twisted boundary condition at $\tau=\beta$ [7]. This is expressed, in the operator formalism, as

$$
Z(\beta, \mu)=\sum_{A} \int[d \xi d \bar{\xi}]\left\langle A, \xi, \bar{\xi}|\mathcal{O}| A,-\sigma \xi,-\sigma^{*} \bar{\xi}\right\rangle,
$$

in which the label $A$ denotes a set of quantum numbers other than the quark occupation and

$$
\begin{gathered}
\langle A, \xi, \bar{\xi}|=\langle A|\left(\bigotimes_{i=1}^{N}\left\langle\xi_{i}, \bar{\xi}_{i}\right|\right), \\
{[d \xi d \bar{\xi}]=d \xi_{1} d \bar{\xi}_{1} \cdots d \xi_{N} d \bar{\xi}_{N},} \\
\mathcal{O}=\mathrm{e}^{-\beta\left(H-\mu \sum_{i=1}^{N}\left(a_{i}^{\ddagger} a_{i}-b_{i}^{\dagger} b_{i}\right)\right)}, \\
\sigma=\mathrm{e}^{i \frac{2 \pi k}{N}} \quad(k \in\{0,1, \ldots, N-1\}) .
\end{gathered}
$$

(For simplicity, the degree of freedom associated with the Dirac spinor components is suppressed.) Applying the relation (A9) and taking account of the commutativity among $H, a_{i}^{\dagger} a_{i}$, and $b_{i}^{\dagger} b_{i}$, one immediately derives the RW periodicity:

$$
\begin{aligned}
Z(\beta, \mu)= & \sum_{A} \int[d \xi d \bar{\xi}] \\
& \times\left\langle A, \xi, \bar{\xi}\left|\mathrm{e}^{-\beta\left(H-\left(\mu+i \frac{2 \pi k}{\beta N}\right) \sum_{i=1}^{N}\left(a_{i}^{\dagger} a_{i}-b_{i}^{\dagger} b_{i}\right)\right)}\right| A,-\xi,-\bar{\xi}\right\rangle \\
= & Z\left(\beta, \mu+i \frac{2 \pi k}{\beta N}\right) .
\end{aligned}
$$

\section{APPENDIX B: PARAMETERS RELATED TO $\psi$ IN $L_{\mathrm{CSC}}$}

In order to understand the role of $\psi$, we consider the equations of motion, which are solved in the Lorentzian space-time in Ref. [14]. For the bulk solution (2) given in Sec. II A, they are given as

$$
\begin{gathered}
\psi^{\prime \prime}+\left(\frac{6}{r}+\frac{f^{\prime}}{f}\right) \psi^{\prime}+\frac{1}{r^{2} f}\left(\frac{q^{2} \phi^{2}}{r^{2} f}-m^{2}\right) \psi=0, \\
\phi^{\prime \prime}+\frac{4}{r} \phi^{\prime}-\frac{2 q^{2} \psi^{2}}{r^{2} f} \phi=0,
\end{gathered}
$$

where $A=A_{\mu} d x^{\mu}=\phi(r) d t$ and $\psi=\psi(r)$ are assumed. ${ }^{4}$

The conformal dimension of the scalar, say $\Delta$, is related to the mass as

$$
\Delta=\frac{1}{2}\left(d+\sqrt{d^{2}+4 m^{2}}\right) .
$$

Here we suppose that the scalar is dual to the Cooper pair, so the dimension is expected to be $\Delta=2 \times \frac{d-1}{2}=d-1$, which is realized for $m^{2}=-(d-1)$. We notice here that $d=5$ and $m^{2}=-4$. Then the asymptotic forms of $\phi$ and $\psi$ are expected as

$$
\phi=\mu-\frac{\bar{d}}{r^{3}}, \quad \psi=\frac{J_{c}}{r}+\frac{C}{r^{4}}, \quad(r \rightarrow \infty),
$$

where $\mu, \bar{d}, J_{\mathrm{c}}$, and $C$ denote the chemical potential, charge density, source, and the vacuum expectation value of the dual operator of $\psi$, respectively.

Here we set the mass of the scalar as $m^{2}=-4$. This is within the Breitenlohner-Friedman (BF) bound, $m^{2}>-d^{2} / 4=-25 / 4$. However, in the present case, the mass is effectively deformed by the background and $\phi$. As a result, the effective mass, which depends on $r$, becomes smaller than the BF bound in some region of $r$. When such a region exists, an instability could occur. To rescue this instability, the condensate of $\psi$ is needed. This is the reason why we could find a nontrivial solution, $\psi \neq 0$. As a result, we find the condensation of the Cooper pair.

In the case of an AdS-Schwarzschild solution, the effective mass is written as

$$
m_{\mathrm{eff}}^{2}=m^{2}-\frac{q^{2} \phi^{2}}{r^{2} f} .
$$

We understand from this relation that, for enough large $q \mu$, we could find the Cooper pair condensation. On the other hand, it would be impossible to realize such a condensation for imaginary $\mu$, since $q^{2} \mu^{2}<0$.

\footnotetext{
${ }^{4}$ We notice that the Euclidean equations are obtained by making the replacement $\phi \rightarrow i \phi$.
} 
[1] S. Kobayashi, D. Mateos, S. Matsuura, R. C. Myers, and R. M. Thomson, Holographic phase transitions at finite baryon density, J. High Energy Phys. 02 (2007) 016.

[2] N. Horigome and Y. Tanii, Holographic chiral phase transition with chemical potential, J. High Energy Phys. 01 (2007) 072.

[3] K. Ghoroku, K. Kubo, M. Tachibana, and F. Toyoda, Holographic cold nuclear matter and neutron star, Int. J. Mod. Phys. A 29, 1450060 (2014).

[4] K. Ghoroku, K. Kubo, M. Tachibana, T. Taminato, and F. Toyoda, Holographic cold nuclear matter as dilute instanton gas, Phys. Rev. D 87, 066006 (2013).

[5] E. Bilgici, F. Bruckmann, C. Gattringer, and C. Hagen, Dual quark condensate and dressed Polyakov loops, Phys. Rev. D 77, 094007 (2008).

[6] E. Bilgici, F. Bruckmann, J. Danzer, C. Gattringer, C. Hagen, E. M. Ilgenfritz, and A. Maas, Fermionic boundary conditions and the finite temperature transition of QCD, Few Body Syst. 47, 125 (2010).

[7] A. Roberge and N. Weiss, Gauge theories with imaginary chemical potential and the phases of QCD, Nucl. Phys. B275, 734 (1986).

[8] G. Aarts, S. P. Kumar, and J. Rafferty, Holographic RobergeWeiss transitions, J. High Energy Phys. 07 (2010) 056.

[9] J. Rafferty, Holographic Roberge-Weiss transitions II: Defect theories and the Sakai-Sugimoto model, J. High Energy Phys. 09 (2011) 087.

[10] H. Isono, G. Mandal, and T. Morita, Thermodynamics of QCD from Sakai-Sugimoto model, J. High Energy Phys. 12 (2015) 006.

[11] F. Bigazzi and A. L. Cotrone, Holographic QCD with dynamical flavors, J. High Energy Phys. 01 (2015) 104.

[12] E. Witten, Anti-de Sitter space, thermal phase transition, and confinement in gauge theories, Adv. Theor. Math. Phys. 2, 505 (1998).

[13] O. Aharony and E. Witten, Anti-de Sitter space and the center of the gauge group, J. High Energy Phys. 11 (1998) 018.

[14] K. Ghoroku, K. Kashiwa, Y. Nakano, M. Tachibana, and F. Toyoda, color superconductivity in holographic SYM theory, Phys. Rev. D 99, 106011 (2019).

[15] K. B. Fadafan, J. C. Rojas, and N. Evans, A holographic description of colour superconductivity, Phys. Rev. D 98, 066010 (2018).

[16] P. Basu, F. Nogueira, M. Rozali, J. B. Stang, and M. Van Raamsdonk, Towards a holographic model of color superconductivity, New J. Phys. 13, 055001 (2011).
[17] M. D'Elia and F. Negro, Theta Dependence of the Deconfinement Temperature in Yang-Mills Theories, Phys. Rev. Lett. 109, 072001 (2012).

[18] G. Endrodi, Z. Fodor, S. D. Katz, and K. K. Szabo, The QCD phase diagram at nonzero quark density, J. High Energy Phys. 04 (2011) 001.

[19] C. Bonati, M. D'Elia, M. Mariti, M. Mesiti, F. Negro, and F. Sanfilippo, Curvature of the chiral pseudocritical line in QCD, Phys. Rev. D 90, 114025 (2014).

[20] C. Bonati, M. D’Elia, M. Mariti, M. Mesiti, F. Negro, and F. Sanfilippo, Curvature of the chiral pseudocritical line in QCD: Continuum extrapolated results, Phys. Rev. D 92 , 054503 (2015).

[21] R. Bellwied, S. Borsanyi, and Z. Fodor, The QCD phase diagram from analytic continuation, Phys. Lett. B 751, 559 (2015).

[22] P. Cea, L. Cosmai, and A. Papa, Critical line of $2+1$ flavor QCD: Toward the continuum limit, Phys. Rev. D 93, 014507 (2016).

[23] K. Kashiwa and A. Ohnishi, Topological deconfinement transition in QCD at finite isospin density, Phys. Lett. B 772, 669 (2017).

[24] K. Kashiwa and A. Ohnishi, Quark number holonomy and confinement-deconfinement transition, Phys. Rev. D 93, 116002 (2016).

[25] T. M. Doi and K. Kashiwa, Dirac-mode expansion of quark number density and its implications of the confinementdeconfinement transition, arXiv:1706.00614.

[26] T. M. Doi and K. Kashiwa, Dirac-mode analysis for quark number density and its application for deconfinement transition, EPJ Web Conf. 175, 12003 (2018).

[27] C. S. Fischer, Deconfinement Phase Transition and the Quark Condensate, Phys. Rev. Lett. 103, 052003 (2009).

[28] K. Kashiwa, H. Kouno, and M. Yahiro, Dual quark condensate in the Polyakov-loop extended NJL model, Phys. Rev. D 80, 117901 (2009).

[29] F. Xu, H. Mao, T. K. Mukherjee, and M. Huang, Dressed Polyakov loop and flavor dependent phase transitions, Phys. Rev. D 84, 074009 (2011).

[30] S. Benić, Physical interpretation of the dressed Polyakov loop in the Nambu-Jona-Lasinio model, Phys. Rev. D 88, 077501 (2013).

[31] Y. Ohnuki and T. Kashiwa, Coherent states of Fermi operators and the path integral, Prog. Theor. Phys. 60, 548 (1978). 